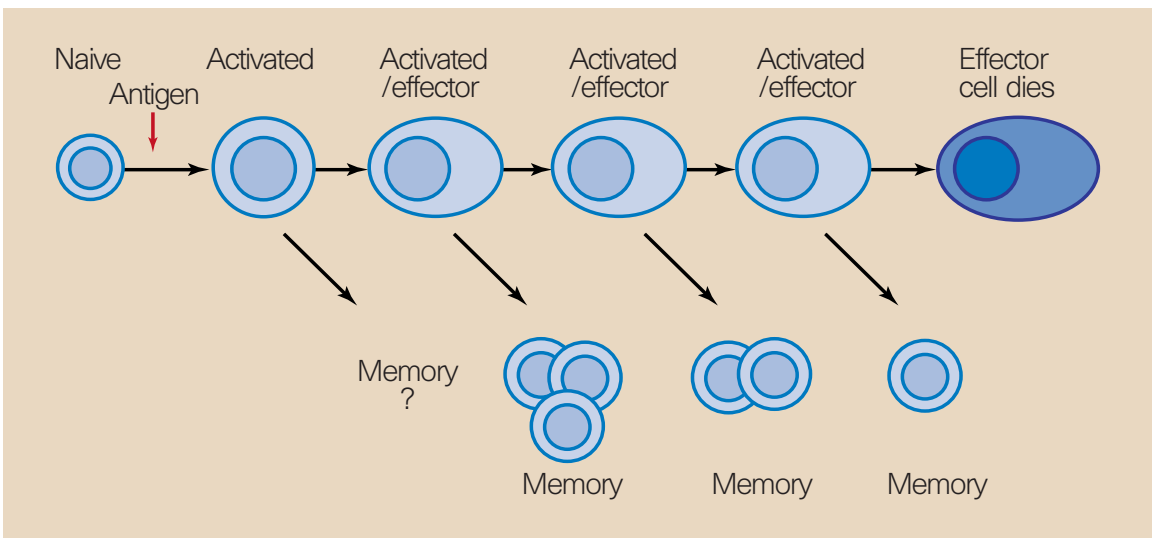

Figure 2 Model for the development of memory T cells. When activated by a specific antigen, $\mathrm{T}$ cells divide and differentiate, and start to carry out effector functions. Such activated/effector cells can revert to memory cells. But successive cell divisions reduce the chances that a $\mathrm{T}$ cell will develop into a memory $\mathrm{T}$ cell, and increase the chances that it will die.

in peripheral areas such as the spleen and lymph nodes express memory markers ${ }^{4}$, yet the authors found no PLAP-containing $\mathrm{T}$ cells in these areas. They found PLAP only in the Peyer's patches (collections of lymphocytes in the wall of the small intestine). To investigate possible differences between the $\mathrm{T}$ cells that expressed PLAP and those that didn't, Jacob and Baltimore studied induction of PLAP expression in a well-characterized model — in vivo infection with lymphocytic choriomeningitis virus (LCMV).

When antigen determinants (specific peptide sequences that the immune system recognizes) are known, antigen-specific $\mathrm{T}$ cells can be identified in vivo. These peptides can be complexed to tetrameric molecules of the major histocompatibility complex, and labelled with fluorochromes. Antigenspecific $\mathrm{T}$ cells then bind to these labelled complexes. This method allows the kinetics of antigen-specific T cells to be followed after immunization, and others ${ }^{5}$ have used it to characterize the immune response after infection with LCMV. In the naive animal, LCMV-specific T cells are so rare they cannot be identified. Viral infection is followed by massive proliferation of these T cells - at the peak of the response, as many as $70-90 \%$ of the total CD8-positive T cells are specific to LCMV. But once the virus has been eliminated, most of these antigen-specific T cells do not survive the contraction phase, with only about $5 \%$ becoming memory $\mathrm{T}$ cells $\mathrm{s}^{5}$.

When following PLAP expression in mice infected with LCMV, Jacob and Baltimore $^{2}$ found that only about $10 \%$ of the LCMV-specific T cells become PLAP-positive during the acute phase of the response. So what is the difference between the LCMV-specific T cells that contain PLAP and those that don't? Both populations seemed to contain effector $\mathrm{T}$ cells, as both could kill target cells, but they differed in their long-term survival. Whereas at least some of the PLAP-containing cells could proliferate in vitro in response to antigen, those that did not contain PLAP simply died. Most importantly, PLAP expression was associated with the ability to become memory T cells - half of the cells that contained PLAP survived in vivo in this form. Although some memory cells lacking PLAP could have formed, most of the $\mathrm{T}$ cells that survived the contraction phase to become memory T cells probably contained PLAP.

Why did only a fraction of the activated $\mathrm{T}$ cells express the transgenic Cre recombinase and produce PLAP? We don't know, but studies of the physiological differences between those T cells that contain PLAP and those that do not will stimulate a new field of research into the differences in T-cell behaviour after they have been stimulated with antigen in vivo. Interestingly, the $\mathrm{T}$ cells that subsequently became memory cells proliferated relatively weakly during the expansion phase of the immune response. This observation supports a model of T-cell differentiation in which increasing cell stimulation and division are associated with progress to terminal differentiation and a reduction in the memory potential (Fig. 2). In other words, cells that divide many times are more likely to die than to survive as memory cells.

Jacob and Baltimore's results indicate that we should now be able to identify T cells with 'memory' potential from among the bulk of antigen-reactive T cells. Effective vaccination depends on the generation of memory cells - so if cells with memory potential express PLAP, this expression may be used to identify efficient immunization protocols. And, if confirmed in other infection models, the approach could be invaluable for assessing immune responses.

Benedita Rocha is at the Institut Necker, INSERM U 345, 156 rue de Vaugirard, 75015 Paris, France. e-mail:rocha@necker.fr

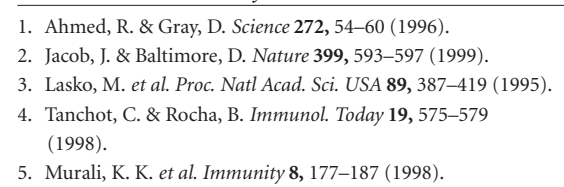

Daedalus

\section{Drawn from life}

Optical and electronic communication uses vast amounts of bandwidth, essentially for pictures. Clever coding can compress this information, but must still retain enough to reconstruct the whole visual extravaganza at the far end. Yet in many cases pictures convey very little of value, even assuming they are necessary at all. Indeed, DREADCO's information scientists are now writing a special NDP ('No Damned Pictures') program for Internet users. It will reject the slowdownloading visual nonsense, while accepting elements of text that actually have something to say.

But even Daedalus regards this as bit extreme. He is looking for a middle way. $\mathrm{He}$ points out that a good cartoonist can capture the essence of a scene with a few well-chosen lines. Why is a line drawing so direct and easy to interpret? Our visual system must work largely on edge detectors; yet this cannot be the whole story. A reversed white-on-black line drawing is not nearly so easy to understand. But clearly a vast amount of bandwidth could be saved if the full bitmap of an image could be reduced to an equivalent line drawing.

So a panel of DREADCO cartoonists is making sketches of a wide variety of photographs, while psychologists study their choice of lines, and programmers strive to embody these choices in software. Their pilot program merely looks for continuous boundaries defining a sharp change in visual contrast. It renders these as black lines on a white ground, and ignores everything else. The results are promising, but imperfect. Block colours, added by an electronic 'painting by numbers' routine, seem to help a bit. An effective 'Videosketch' program will need much greater insight and subtlety; but the thing seems possible.

When perfected, Videosketch will transform business and scientific communication. It will transmit useful material, such as text, drawings, and graphs, almost unchanged. Rococo visual detail, however, will be drastically pruned. Photographs will become sketches, video clips will become cartoon films, videoconferencing will acquire an intriguing surreality. Even entertainment may benefit. Daedalus will be interested to see if video on demand, that great white hope of the cable industry, takes off in cartoon form. 'Adult entertainment' might even gain from the stark, compelling simplicity of its Videosketch representation.

David Jones 